\title{
HADEC: Hadoop-based live DDoS detection framework
}

\author{
Sufian Hameed ${ }^{*}$ (D) and Usman Ali
}

\begin{abstract}
Distributed denial of service (DDoS) flooding attacks are one of the main methods to destroy the availability of critical online services today. These DDoS attacks cannot be prevented ahead of time, and once in place, they overwhelm the victim with huge volume of traffic and render it incapable of performing normal communication or crashes it completely. Any delays in detecting the flooding attacks completely halts the network services. With the rapid increase of DDoS volume and frequency, the new generation of DDoS detection mechanisms are needed to deal with huge attack volume in reasonable and affordable response time.

In this paper, we propose HADEC, a Hadoop-based live DDoS detection framework to tackle efficient analysis of flooding attacks by harnessing MapReduce and HDFS. We implemented a counter-based DDoS detection algorithm for four major flooding attacks (TCP-SYN, HTTP GET, UDP, and ICMP) in MapReduce, consisting of map and reduce functions. We deployed a testbed to evaluate the performance of HADEC framework for live DDoS detection on low-end commodity hardware. Based on the experiment, we showed that HADEC is capable of processing and detecting DDoS attacks in near to real time.
\end{abstract}

Keywords: DDoS, Flooding attacks, DDoS detection, Hadoop

\section{Introduction}

DDoS flooding attacks are one of the biggest threats faced by the critical IT infrastructure, from the simplest enterprise network to complex corporate networks. DDoS attacks are here since the very advent of computer networks, and they are not going anywhere anytime soon. The first DDoS attack incident [17] was reported in 1999 by the Computer Incident Advisory Capability (CIAC). Since then, most of the DoS attacks are distributed in nature and they continue to grow in frequency, sophistication, and bandwidth. The main aim of these attacks is to overload the victim's machine and make his services unavailable, leading to revenue losses.

Over the years, DDoS has hit major companies and Internet infrastructures, incurring significant loss in revenues. Yahoo! experienced one of the first major DDoS flooding attacks that made their services offline for about $2 \mathrm{~h}$ [14]. In October 2002, 9 of the 13 domain name system (DNS) root servers were shut down for an hour because of a DDoS flooding attack [10]. During the fourth quarter

*Correspondence: sufian.hameed@nu.edu.pk

IT Security Labs, National University of Computer and Emerging Sciences

(NUCES), Karachi, Pakistan of 2010, a hacktivist group called Anonymous orchestrated major DDoS flooding attacks and brought down the Mastercard, PostFinance, and Visa websites [9]. Most recently, online banking sites of nine major US banks (i.e., Bank of America, Citigroup, Wells Fargo, U.S. Bancorp, PNC, Capital One, Fifth Third Bank, BB\&T, and HSBC) have been continuously the targets of powerful DDoS flooding attack series [17]. The legacy of DDoS continue to grow in sophistication and volume with recent attacks breaking the barrier of hundreds of Gbps [41].

On 21 October 2016, IoT devices (such as printers, cameras, home routers, and baby monitors) were used to generate DDoS attack involving malicious DNS lookup requests from tens of millions of IP addresses [1]. This attack, at that time, was considered largest of its kind in the history with an unprecedented rate of $1.2 \mathrm{Tbps}$. The main target of the attack was the servers of DYN Inc., a company that controls much of the Internet's DNS infrastructure [25]. This attack was carried out multiple times which rendered major Internet platforms and services unavailable to large swaths of users in Europe and North America for several hours throughout the day.

\section{Springer Open}

(C) The Author(s). 2018 Open Access This article is distributed under the terms of the Creative Commons Attribution 4.0 International License (http://creativecommons.org/licenses/by/4.0/), which permits unrestricted use, distribution, and reproduction in any medium, provided you give appropriate credit to the original author(s) and the source, provide a link to the Creative Commons license, and indicate if changes were made. 
Study of recent attacks reveals that with little effort, next generation attack tools would be able to enact DDoS attacks that are thousand times stronger than the ones we see today [33]. One of the major concerns is that performing DDoS attack is extremely simple with websites known as Booters or Stressers that offer DDoS as a service. These booters provide cheap services, and the costs to perform a series of attacks is typically just a few dollars [36]. Recently, GitHub was hit by 1.35 Tbps of traffic all at once [2]. It was the most powerful attack in the history of DDoS, and the alarming fact is that no botnet was required to achieve such high traffic volume. The attackers spoofed GitHub's IP addresses and took control of its memcached (a distributed memory system known for high performance and demand) instances. The memcached systems then return 50 times the data of the requests back to the victim. GitHub called Akamai Prolexic for DDoS mitigation service, and the assault dropped off after $8 \mathrm{~min}$. GitHub was lucky enough to afford robust DDoS mitigation services, but for small to medium enterprises and financial institutions, there is a need for new low cost DDoS defense mechanisms and architectures that can be easily deployed on commodity hardware.

The explosive increase in the volume of Internet traffic and sophistication of DDoS attacks have posed serious challenges on how to analyze the DDoS attacks in a scalable and accurate manner. For example, two of the most popular open-source intrusion detection systems (IDS), Snort [35] and Bro [32], maintain per-flow state to detect anomalies. The Internet traffic doubles every year, and due to that monitoring, large amount of traffic in real-time anomaly detection with conventional IDS has become a bottleneck. Existing solutions require a lot of resource and do not add any value to limited resource environment. Therefore, we need new solutions that can detect DDoS attack efficiently in near to real time.

In [27], Lee et al. have proposed a DDoS detection method based on Hadoop [3]. They have used a Hadoopbased packet processor [26] and devised a MapReduce [7]based detection algorithm against the HTTP GET flooding attack. They employ a counter-based DDoS detection algorithm in MapReduce that counts the total traffic volume or the number of web page requests for picking out attackers from the clients. For experiments, they used multiple Hadoop nodes (max. 10) in parallel to show the performance gains for DDoS detection. Unfortunately, their proposed framework, in its current form, can only be used for offline batch processing of huge volume of traces. The problem to develop a real time defense system for live analysis still needs to be tackled.

In this paper, we propose HADEC, a Hadoop-based live DDoS detection framework. HADEC is a novel destination-based DDoS defense mechanism that leverages Hadoop to detect live DDoS flooding attacks in wired networked systems. The real motivation behind this study is to check the efficacy of scalable defenses against DDoS flooding attacks using new distributed architectures that can run on low cost commodity hardware. This will help small and medium organizations and financial institutes to protect their infrastructure with in-house low-cost solutions. HADEC comprises of two main components, a capturing server and a detection server. Live DDoS starts with the capturing of live network traffic handled by the capturing server. The capturing server then processes the captured traffic to generate log file and transfer them to the detection server for further processing. The detection server manages a Hadoop cluster, and on the receipt of the log file(s), it starts MapReduce-based DDoS detection jobs on the cluster nodes. The proposed framework implements a counter-based algorithm to detect four major DDoS flooding attacks ${ }^{1}$ (TCP-SYN, UDP, ICMP, and HTTP GET). These algorithms execute as a reducer job on the Hadoop detection cluster.

An early version of this work appeared as a short paper [21] and also as a technical report at arXiv [23]. In this paper, we provide a more detailed illustration of different HADEC components. We have added significant new results (approx. 50\% new) with different attack volumes, i.e., $80-20$ (80\% attack traffic and $20 \%$ legitimate traffic) and $60-40(60 \%$ attack traffic and $40 \%$ legitimate traffic). 80-20 traffic volume is used to emulate flooding behavior where attack traffic surpasses the legitimate one, whereas 60-40 traffic volume is used to emulate low volume attacks where legitimate and attack traffic volume is relatively close. We also performed system benchmarks to show the overall CPU and memory utilized by HADEC during different phases of data capturing, transfer, and attack detection. These system benchmarks are important to evaluate how well the proposed solution works on low-end commodity hardware under different stress conditions.

We deploy a testbed for HADEC on commodity hardware (low-end desktop machine comprising of core i5 $\mathrm{CPU})$, which consists of a capturing server, detection server, and a cluster of ten physical machines, each connected via a Gigabit LAN. We evaluate HADEC framework for live DDoS detection by varying the attack volume and cluster nodes. HADEC is capable of analyzing $20 \mathrm{~GB}$ of $\log$ file, generated from 300 GBs of attack traffic, in approx. 8.35 mins on a cluster of 10 nodes. For small log files representing $1.8 \mathrm{Gbps}$, the overall detection time is approximately $21 \mathrm{~s}$. Our system benchmark evaluations show that during the packet capture phase, the CPU usage is $50 \%$ on average, whereas the CPU usage remained low with an average of $15 \%$ during the log transfer phase. The capturing phase remains low on memory usage with a constant at around 1000 MBs (12-13\%), while the transfer phase consumes on average 7600 MBs (94\%). The 
benchmarks on Hadoop's NameNode show reduction in CPU usage with the increase in cluster size and parallelism.

The rest of the paper is organized as follows. Section 2 describes the state of the art. Section 3 describes the HADEC framework design. In Section 4, we discuss the testbed deployment. Section 5 demonstrates the performance of the proposed framework. Comparison with existing work and optimization recommendation for HADEC deployment is discussed in Section 6. Finally, we conclude the paper in Section 7.

\section{Related work}

Since the inception of DDoS flooding attacks, several defense mechanisms have been proposed to date in the literature [41]. This section highlights the DDoS flooding attacks, followed by a discussion on the application of Hadoop to combat network anomalies, Botnet- and DDoS-related attacks.

The DDoS flooding attacks can be categorized into two types based on the protocol level that is targeted: network/transport-level attacks (UDP flood, ICMP flood, DNS flood, TCP SYN flood, etc.) and applicationlevel attacks (HTTP GET/POST request). The defense mechanisms against network or transport-level DDoS flooding attacks roughly falls into four categories: source-based, destination-based, network-based, and hybrid (distributed), and the defense mechanisms against application-level DDoS flooding attacks have two main categories: destination-based and hybrid (distributed). Since the application traffic is not accessible at the layer 2 and layer 3, there is no network-based defense mechanism for the application-level DDoS. Following is the summary of features and limitations for the DDoS defense categories.

- Source-based: In source-based defense mechanism, the detection and response are deployed at the source hosts in an attempt to mitigate the attack before it wastes lots of resources $[28,29]$. Accuracy is a major concern in this approach as it is difficult to differentiate legitimate and DDoS attack traffic at the sources with low volume of the traffic. Further, there is low motivation for deployment at the source ISP due to added cost for community service.

- Destination-based: In this case the detection and response mechanisms are deployed at the destination hosts. Access to the aggregate traffic near the destination hosts makes the detection of DDoS attack easier and cheaper, with high accuracy, than other mechanisms $[34,37,38]$. On the downside, destination-based mechanisms cannot preempt a response to the attack before it reaches the victim and wastes resources on the paths to the victim.
- Network-based: With network-based approach, the detection and response are deployed at the intermediate networks (i.e., routers). The rationale behind this approach is to filter the attack traffic at the intermediate networks and as close to source as possible [30, 31]. Network-based DDoS defenses incur high storage and processing overhead at the routers, and accurate attack detection is also difficult due to lack of sufficient aggregated traffic destined for the victims.

- Hybrid (Distributed): In hybrid approach, there is coordination among different network components along the attack path and detection and response mechanisms are deployed at various locations. Destination hosts and intermediate networks usually deploy detection mechanisms, and response usually occurs at the sources and the upstream routers near the sources $[39,40]$. Hybrid approach is more robust against DDoS attacks, but due to distributed nature, it requires more resources at various levels (e.g., destination, source, and network) to tackle DDoS attacks. The complexity and overhead because of the coordination and communication among distributed components is also a limiting factor in smooth deployment of hybrid-based DDoS defenses. Recent advents in software defined networking (SDN) bring us new approaches to deal with DDoS attacks in a collaborative manner. SDN controllers lying in different autonomous systems (AS) can securely communicate and transfer attack information with each other. This enables efficient notification along the path of an ongoing attack and effective filtering of trafic near the source of attack, thus saving valuable time and network resources [22, 24].

Analysis of logs and network flows for anomaly detection has been a problem in the information security for decades. New big data technologies, such as Hadoop, has attracted the interest of the security community for its promised ability to analyze and correlate securityrelated heterogeneous data efficiently and at unprecedented scale and speeds [15]. In the rest of the section, we review some recent techniques (other than [27] , discussed in Section 1) where Hadoop-based frameworks are used to build affordable infrastructures for security applications.

BotCloud [19] propose a scalable P2P detection mechanism based on MapReduce and combination of host and network approaches [20]. First, they generate large dataset of Netflow data [16] on an individual operator. Next, they applied a PageRank algorithm on the Netflow traces to differentiate the dependency of hosts connected in P2P fashion for the detection of botnets. They moved the PageRank algorithm to MapReduce, and the PageRank 
algorithm executes on data nodes of Hadoop cluster for efficient execution.

Temporal and spatial traffic structures are essential for anomaly detectors to accurately drive the statistics from network traffic. Hadoop divides the data into multiple same size blocks and distributes them in a cluster of data nodes to be processed independently. This could introduce a difficulty in analysis of network traffic where related packets may be spread across different block, thus dislocating traffic structures. Hashdoop [18] resolves this potential weakness by using hash function to divide traffic into blocks that preserve the spatial and temporal traffic structures. In this way, Hashdoop conserves all the advantages of the MapReduce model for accurate and efficient anomaly detection of network traffic.

\section{Hadoop DDoS detection framework}

This section gives insights about the working of our proposed framework. The framework is fully automated, which captures the log, transfers them to the Hadoop detection cluster, and starts the detection automatically. The Hadoop-based live DDoS detection framework (HADEC) comprise of four major phases (see Fig. 1). By using this approach, we are able to detect DDoS flooding attack in close to real time.

1. Network traffic capturing and log generation.

2. Log transfer.

3. DDoS detection.

4. Result notification.

Each of the abovementioned phases is implemented as separate components that communicate with each other to perform their assigned task. Traffic capturing and log generation are handled at the capturing server, whereas DDoS detection and result notification are performed by

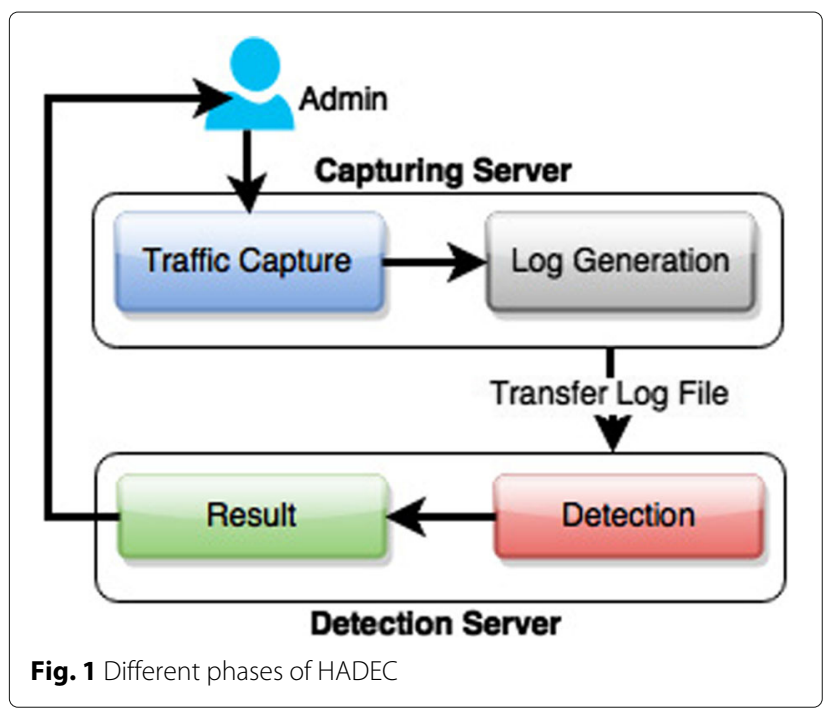

the detection server. Log transfer is handled through web services. In the following subsections, we have explained the functionalities for each of the phase/component in detail.

\subsection{Traffic capturing and log generation}

Live DDoS detection starts with the capturing of network traffic. HADEC provides a web interface through which the admin can tune the capturing server with desired parameters. These parameters are file size, number of files to be captured before initializing the detection phase, and the path to save the captured file. Once the admin is done with the configurations, the Traffic Handler sends the property file to the Echo Class (a java utility to generate logs) and starts the capturing of live network traffic (see Fig. 2).

HADEC use the Tshark library [13] to capture live network traffic. Tshark is an open source library capable of capturing huge amount of traffic. Under default settings, Tshark library runs through command line and outputs the result on console. To log the traffic for later use, we developed a java-based utility (Echo Class) to create a pipeline with Tshark and read all the output packets from Tshark. We have also tuned Tshark to output only the relevant information required during detection phase. This includes information of timestamps, source IP, dst IP, packet protocol, and brief packet header information. The

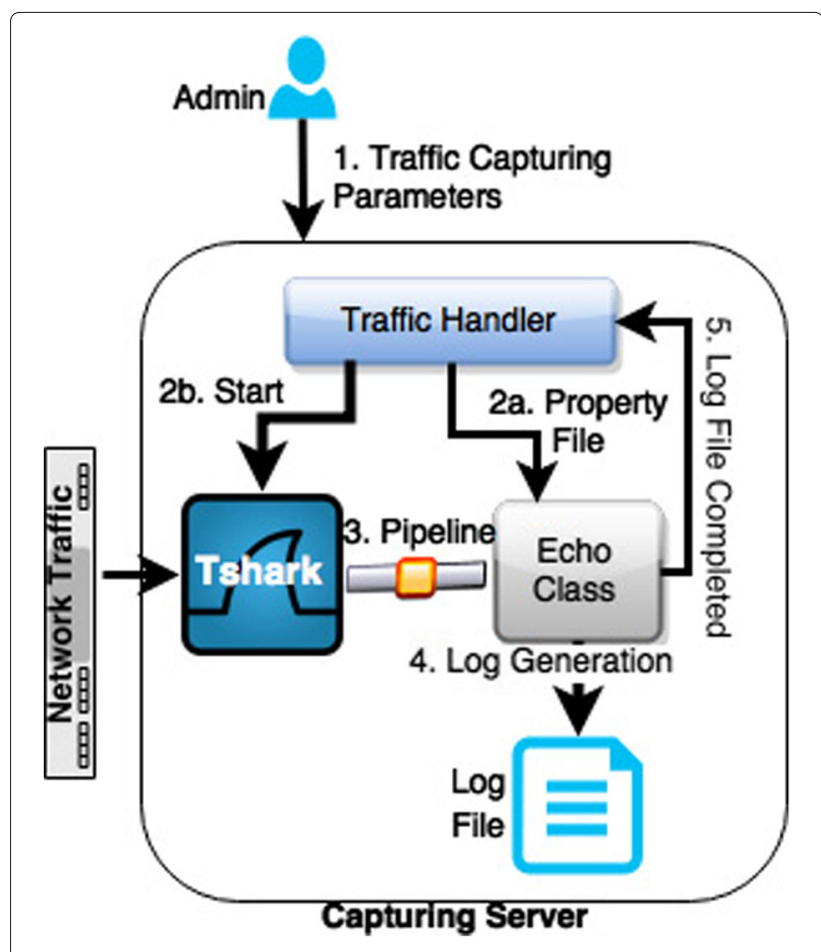

Fig. 2 Network traffic capturing and log generation component 
following are the snippets for TCP (SYN), HTTP, UDP, and ICMP packets that are logged in the file.

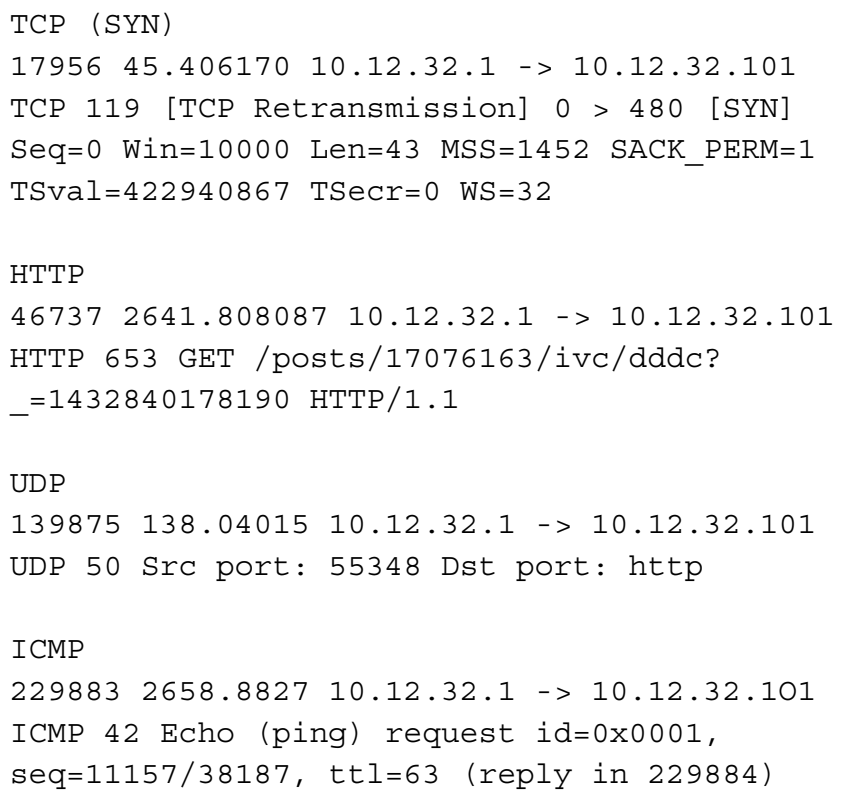

As discussed above, the Traffic Handler sends the property file to the Echo Class with the desired set of parameters (file size, file count for detection, and storage path on the capturing server) set by the admin. Echo Class uses these parameters to generate a log file, at the specified location, when it reads the required amount of data from Tshark. Once the log file is generated, the Echo Class also notifies the Traffic Handler (see Fig. 2).

\subsection{Log transfer phase}

After the $\log$ file is generated, the Traffic Handler will notify the detection server and also share the file information (file name, file path, server name, etc.) with it via a webservice. The detection server will initiate a Secure Copy or SCP protocol [12] (with pre-configured credentials) with the capturing server and transfer the log file from the capturing server (using the already shared name/path information) into its local file system (see
Fig. 3). The capturing server has two network interfaces, one for incoming traffic and one to communicate with the detection server.

Since the detection server mainly works as a NameNode, i.e., the centerpiece of the Hadoop cluster and HDFS (Hadoop distributed file system), it has to transfer the log file(s) from local storage to HDFS. On successful transfer of $\log$ file into HDFS, the detection server sends a positive acknowledgement to the capturing server and both the servers delete that specific file from their local storage to maintain healthy storage capacity. Before starting the DDoS detection process, the detection server will wait for the final acknowledgment from the capturing server. This acknowledgement validates that the desired number of files of a particular size (set via parameters by admin) has been transferred to HDFS before the execution of MapReduce-based DDoS detection algorithm. There is no particular restriction on the minimum file count before the detection starts; it could be set to one.

\subsection{Detection phase}

The Apache Hadoop consists of two core components, i.e., HDFS (storage part) and MapReduce (processing part). Hadoop's central management node also known as NameNode splits the data into same size large blocks and distributes them among the cluster nodes (data nodes). Hadoop MapReduce transfers packaged code for nodes to process in parallel, the data each node is responsible to process.

In HADEC, the detection server mainly serves as the Hadoop's NameNode, which is the centerpiece of the Hadoop DDoS detection cluster. On successful transfer of $\log$ file(s), the detection server splits the file into same size blocks and starts MapReduce DDoS detection jobs on cluster nodes (see Fig. 4). We have discussed MapReduce job analyzer and counterbased DDoS detection algorithm in Section 3.5. Once the detection task is finished, the results are saved into HDFS.

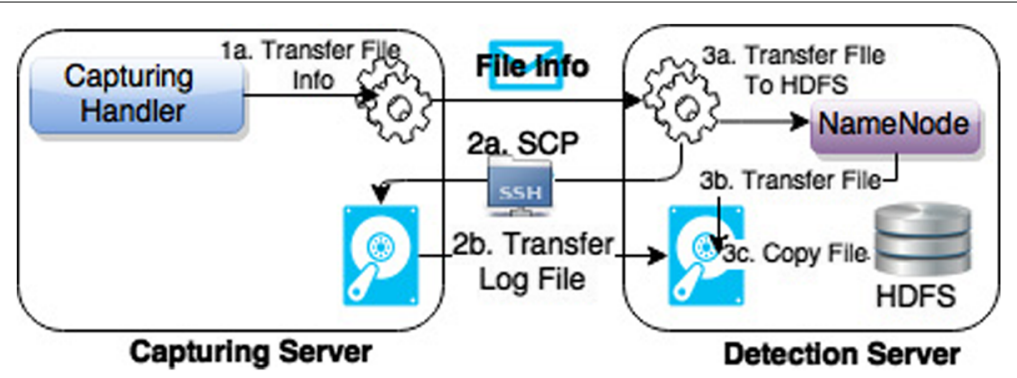

Fig. 3 Log transfer phase 


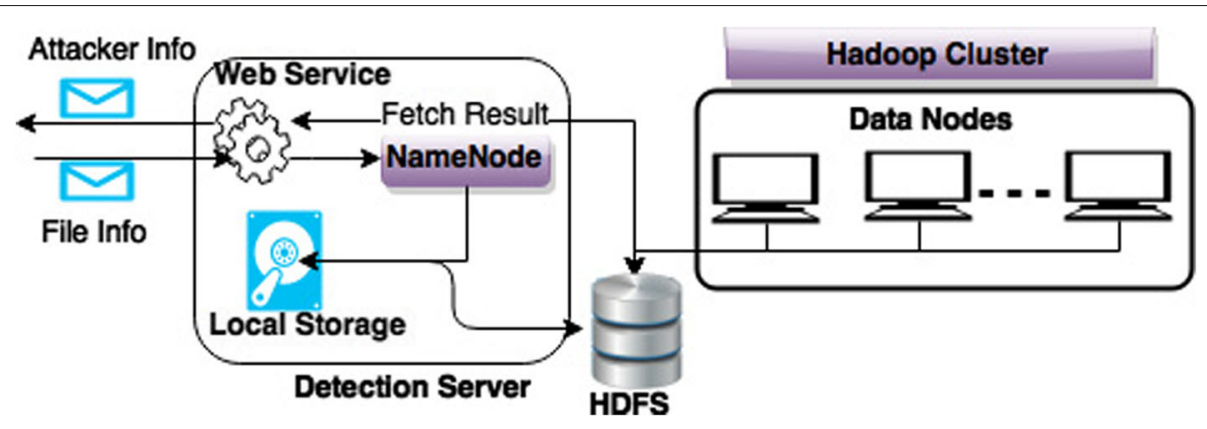

Fig. 4 DDoS detection on Hadoop cluster

\subsection{Result notification}

Once the execution of all the MapReduce tasks is finished, Hadoop will save the results in HDFS. The detection server will then parse the result file from HDFS and send the information about the attackers back to the administrator via the capturing server. Once the results are notified, both the input and output folders from HDFS will be deleted for better memory management by the detection server. Figure 5 presents a holistic illustration of HADEC framework.

\subsection{MapReduce job and DDoS detection}

A MapReduce program is composed of a map task that performs filtering and sorting and a reduce task that performs a summary operation. Here, we have explained how HADEC has implemented detection of DDoS flooding

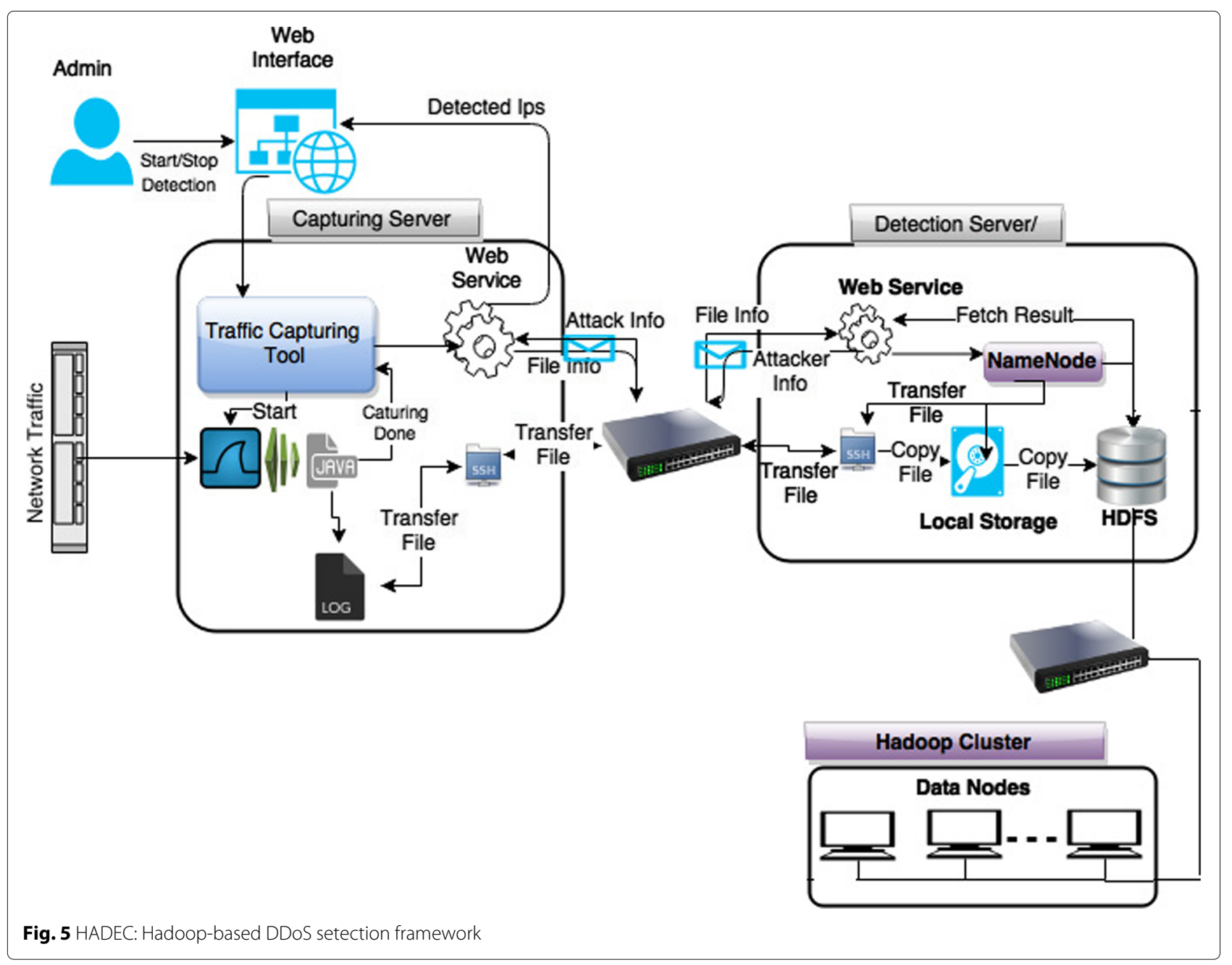


attacks (UDP, HTTP GET, ICMP, and TCP-SYN) as a MapReduce task on Hadoop cluster using counter-based algorithm.

\subsubsection{HADEC mapper job}

After starting MapReduce task, the first task is a mapper task which takes input from HDFS as a block. In our case, the block will represent a file in text format and the input for each iteration of mapper function will be a single line from the file. Any single line in the file contains only brief information of a network packet captured through Tshark (see Section 3.1). The term network packet used in the rest of this section represents a single line content of the file read as a mapper input.

Mapper job takes pair of data as input and returns a list of pairs (key, value). Mapper output type may differ from mapper's input type; in our case, the input of mapper is pair of any number $i$ and the network packet. The output is a list of pair (key, value) with key as the source IP address and value as a network packet. Mapper job also use hashing for combining all the logs of data on the basis of source IP address, so that it becomes easier for reducer to analyze the attack traffic.

After all the mapper have finished their jobs, the data or worker nodes perform a shuffle step. During shuffling, the nodes redistribute the data based on the output keys, such that all data belonging to one key are located on the same worker node (see Fig. 6).

In HADEC, for analysis and detection of UDP flooding attack, the mapper task filters out the packets having UDP information. In particular, the mapper function will search packets having QUIC / UDP information. QUIC stands for Quick UDP Internet connection. For the packet that contains the desired information, the mapper function generates an output in the form of pairs (key, value). The pseudocode for mapper function is as follows.

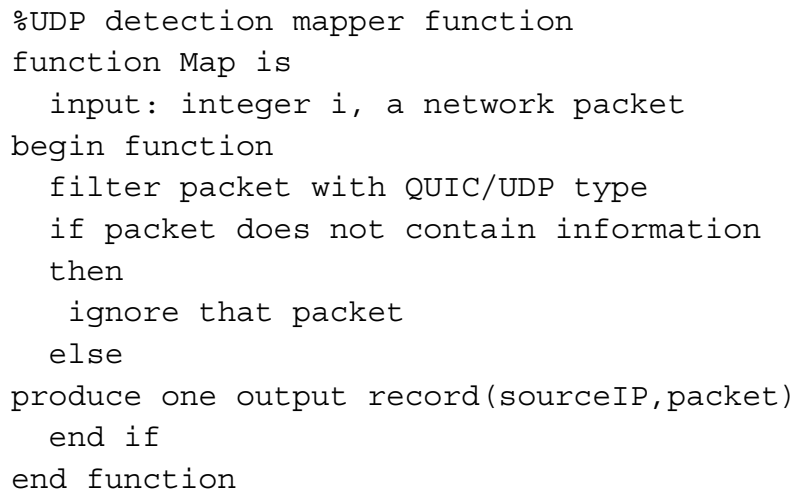

For ICMP-, TCP-SYN-, and HTTP-GET-based flooding attacks, the mapper function will search for SYN, ICMP, and HTTP-GET packet type information respectively.

\subsubsection{HADEC reducer job and counter-based algorithm}

Once the mapper tasks are completed, the reducer will start operating on the list of key/value pairs (i.e., IP/packet pairs) produced by the mapper functions. The reducers are assigned a group with unique key; it means that all the packets with unique key (unique source IP in our case) will be assigned to one reducer. We can configure Hadoop to run reducer jobs on varying number of data nodes. For efficiency and performance, it is very important to identify the correct number of reducers required for finalizing the analysis job. HADEC runs counter-based algorithm to detect DDoS flooding attacks on reducer nodes. The reducer function takes input in key/value pair (source IP, packet of type $\mathrm{X}$ ) and produces a single key/value pair (source IP, no. of packets of type X) output after counting the number instance (see Fig. 6).

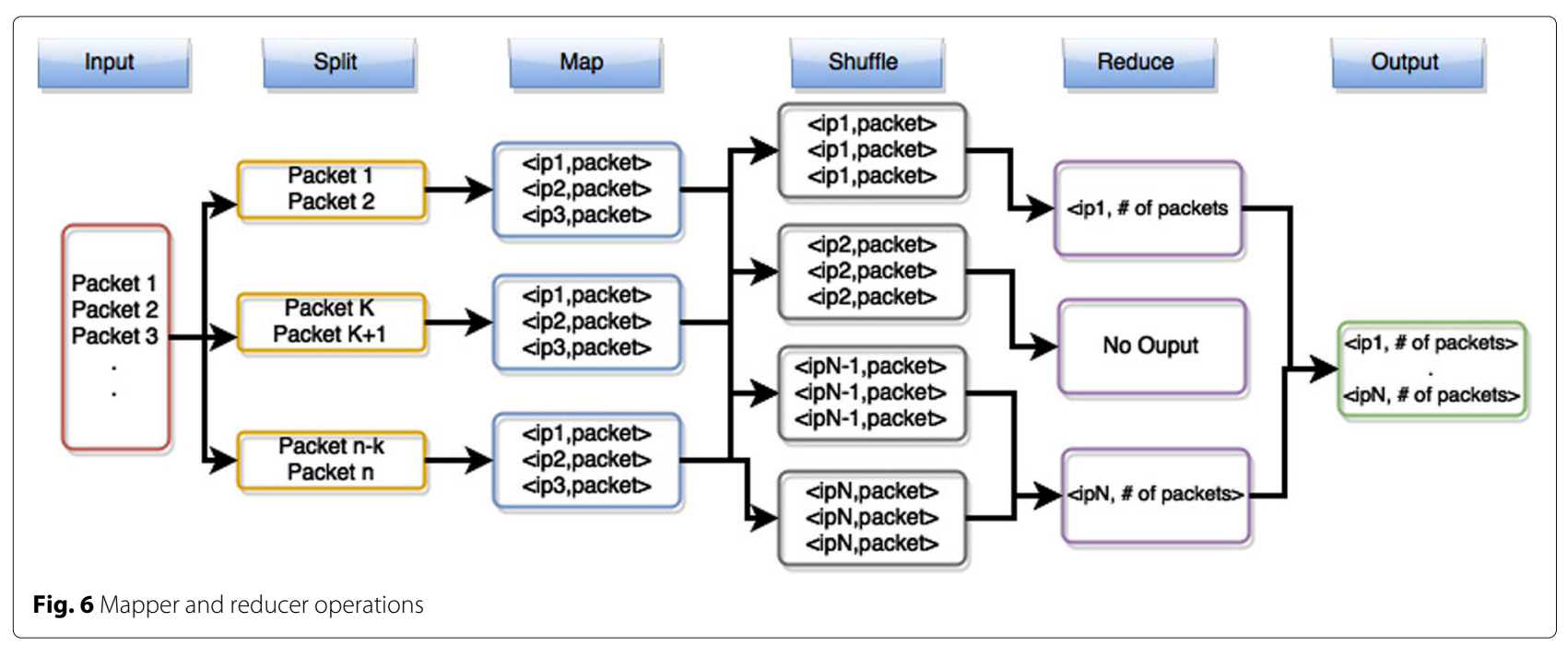


Counter-based algorithm is the simplest, yet very effective algorithm to analyze the DDoS flooding attacks by monitoring the traffic volumes for source IP addresses. The algorithm counts all the incoming packets, of a particular type (UDP, ICMP, HTTP ...etc.), associated with a unique IP address in a unit time. If the traffic volume or count for source IP exceeds the pre-defined threshold, that particular IP will be declared as an attacker. The pseudocode for reducer function using counter-based algorithm for UDP attack is as follows.

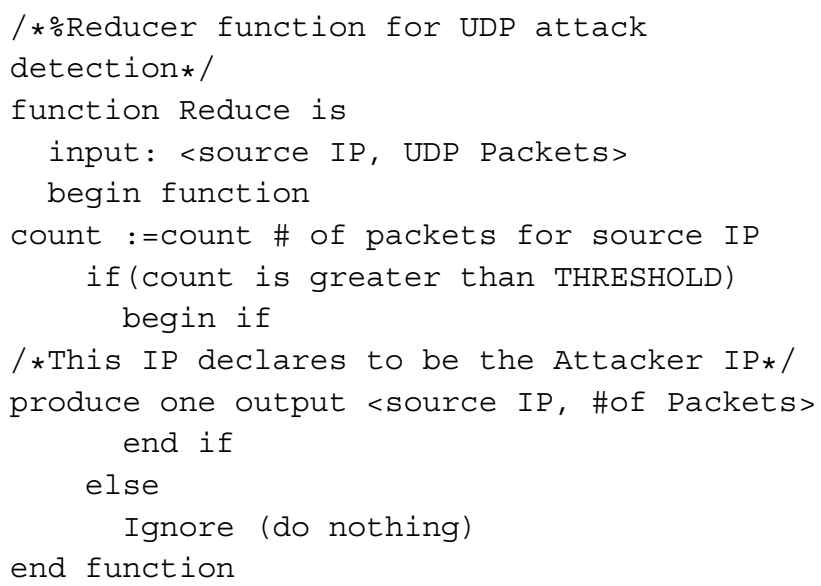

\section{HADEC testbed}

In this section, we have discussed the testbed deployment of HADEC and how we have evaluated the performance of the proposed framework with different experiments.

HADEC performs two main tasks, (a) capturing and transfer of network traffic and (b) detection of DDoS flooding attacks. For capturing the traffic, we use a single node capturing server to capture, process, and send the network traffic to detection server. For DDoS detection, we deploy a single node detection server (also acts as NameNode of Hadoop cluster) and a Hadoop detection cluster consisting of ten nodes. Each node in our testbed (one capturing server, one detection server, and ten Hadoop data nodes) consists of $2.60 \mathrm{GHz}$ Intel core i5 CPU, 8 GB RAM, 500 GB HDD, and 1 Gbps Ethernet card. All the nodes in HADEC used Ubuntu 14.04 and are connected over a Gigabit LAN. We have used Hadoop version 2.6.0 for our cluster and YARN [4] to handle all the JobTracker and TaskTracker functionality.

There are several attack generation tools that are available online, such as LOIC [6], Scapy [11], Mausezahn [8], Iperf [5], etc. For our testbed evaluations, we have mainly used Mausezahn, because of its ability to generate huge amount of traffic with random IPs to emulate different number of attackers. We deployed three dedicated attacker nodes along with couple of legitimate users to flood the victim machine (capturing server) with a traffic volume of up till $913 \mathrm{Mbps}$ (practically highest possible for a Gigabit LAN). HADEC testbed is shown in Fig. 7. For evaluations, we have only focused on UDP flooding attack due to its tendency to reach high volume from limited number of hosts. Any given measurement or datapoint in the result graphs is an average of five readings. We would also like to add that for all the evaluations, we have used only a single reducer, different variations were tried but there was no performance gains.

\section{Performance evaluation}

We have considered different factors for the performance evaluation of our proposed framework. The overall performance of HADEC depends on:

- The time taken for capturing and transferring the log files.

- The number of attackers and attack volume.

- The execution time of DDoS detection algorithm on the Hadoop cluster.

- The system benchmarks (CPU and memory usage).

For our evaluations, we varied different parameters like log file size, Hadoop cluster size, attack volume, Hadoop splits or block size, and threshold for counter-based algorithm, and measured their impact on the performance of HADEC. The admin can change these parameters on the fly in the configuration settings to adjust any errors or bias present in the results. These parameters are briefly described as follows.

- File size: We evaluate the performance of our framework with different sizes of log files. We consider log file size of $10 \mathrm{MB}, 50 \mathrm{MB}, 100 \mathrm{MB}$, $200 \mathrm{MB}, 400 \mathrm{MB}, 600 \mathrm{MB}, 800 \mathrm{MB}$, and $1 \mathrm{~GB}$ and evaluate how well the framework performs with varying $\log$ size.

- Cluster size: Hadoop distributes the processing among the available cluster nodes (datanodes). This means that increase in the cluster size will effect detection phase performance. We used varying cluster size between $2,4,6,8$, and 10 datanodes to observe the performance enhancement.

- Attack volume: For our evaluations, we used two with different attack volumes, i.e., $80-20$ (80\% attack traffic and 20\% legitimate traffic) and 60-40 (60\% attack traffic and 40\% legitimate traffic). 80-20 traffic volume is used to emulate flooding behavior where attack traffic surpasses the legitimate one, whereas 60-40 traffic volume is used to emulate low volume attacks where legitimate and attack traffic volume is relatively close.

- Block size: Hadoop's NameNode splits the log file data into same size large blocks and distributes them among the cluster nodes (data nodes). We evaluated HADEC with different block sizes since they directly influence the degree of parallelism. 


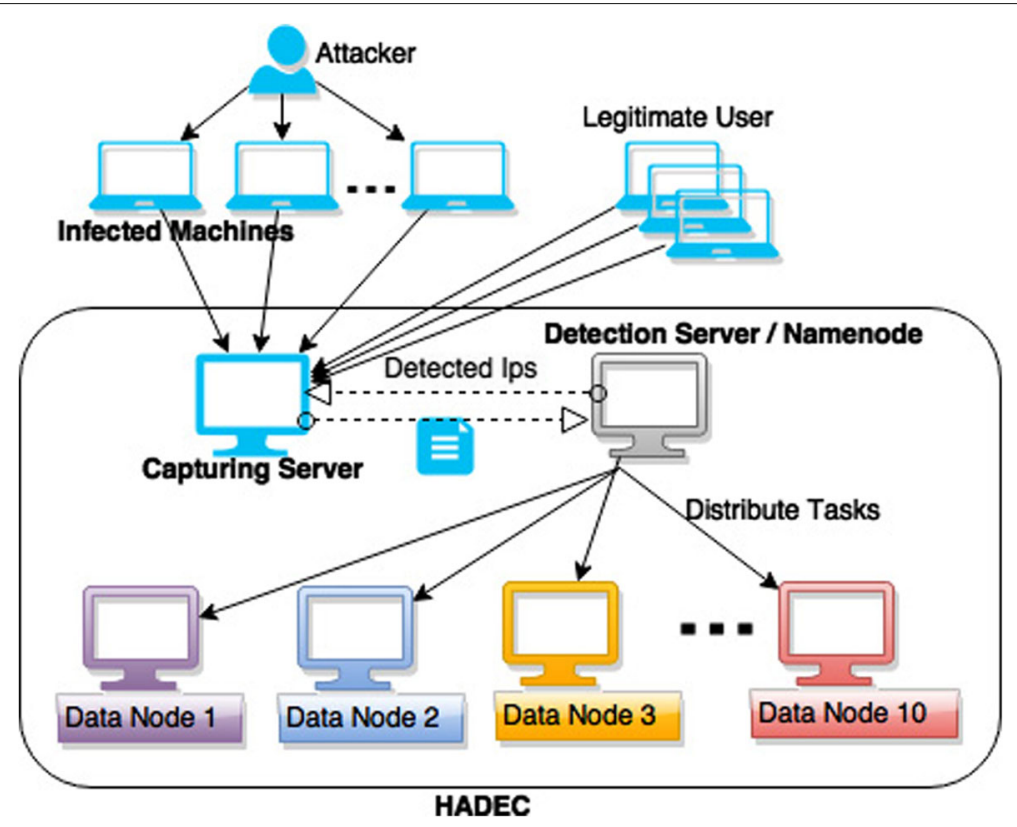

Fig. 7 HADEC testbed

- Threshold: This marks the cutoff frequency after which any particular IP is marked as attacker. We have used two threshold values of 500 and 1000 packets per second for our evaluations.

\subsection{Traffic capturing and file transfer}

The capturing server works on two major tasks simultaneously. First, it captures huge amount of network traffic (913 Mbps in our testbed) and transforms it into log file(s). Second, it transfers the log file(s) to the detection server for further processing. This simultaneous execution of capture and transfer operations are important for live analysis of DDoS flooding attack, but on the other hand, both the operations compete for resources.

Figure 8 shows the capturing and transfer time taken by the capturing server for log files of different sizes. The capturing time is almost linear to the increase in file size. It takes approximately $2 \mathrm{~s}$ to $\log$ a file of $10 \mathrm{MB}$ and extends to $142 \mathrm{~s}$ for $1 \mathrm{~GB}$ file. File transfer takes $14 \mathrm{~s}$ to transfer $10 \mathrm{MB}$ file and approx. $35 \mathrm{~s}$ for $1 \mathrm{~GB}$ file. This shows a clear improvement in throughput with the increase in file size. Here, it is also interesting to note that the transfer operation has to compete for bandwidth, and during peak time, more than $90 \%$ of the bandwidth is being consumed by the

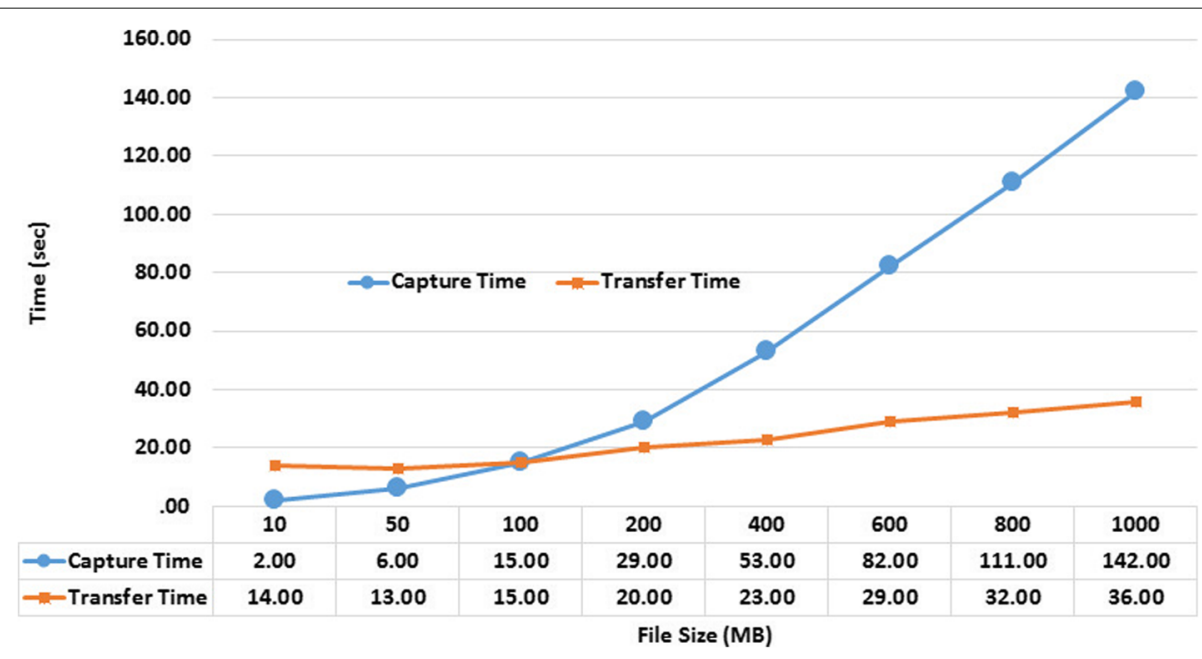

Fig. 8 Capture and transfer time of a log file (file size is in MBs and time is in seconds) 
attack traffic. We already discussed above that all the evaluation results are an average of five readings. However, we rounded the average to whole number in Fig. 8. This is because the unit of evaluation is in seconds and we had clear difference in results for different parameters. For all the remaining results, we kept the value till two decimal places because at time it is hard to observe any difference between different experimental settings.

\subsection{Number of attackers and attack volume}

HADEC use counter-based algorithm to detect attackers. This means that during the DDoS flooding attack, any particular attacker has to cross certain volume threshold to be detected. It is imperative to study the number of attackers one file can capture in it. Table 1 presents the relationship between the size of log file with the total number of attackers and the aggregate traffic volume. According to Table 1, the capturing server has to analyze approx. 0.24 GBs of network traffic to generate a log file of $10 \mathrm{MB}$ and it could represent 100 plus attackers that cross the flooding frequency threshold of 500-1000 packet. By increasing the log file size, the capability to capture accurate information related to attackers also increases. There is a trade-off between the log file size and overall detection rate; therefore, the admin will have to adjust the framework parameters that will best fit in different attack scenarios.

\subsection{DDoS detection on Hadoop cluster}

In this section, we evaluate the performance of DDoS detection on the Hadoop cluster. For our evaluations, we used different sizes of the $\log$ files $(10,50,100,200$, $400,600,800$, and $1000 \mathrm{MBs}$ ), different threshold values (500 and 1000) for counter-based detection algorithm, and attack volume of $80-20$ and $60-40$. For all the evaluations in this section, we used fix Hadoop data block of $128 \mathrm{MB}$.

Figure 9 shows the detection time on Hadoop cluster with 500 threshold. With the increase in file size, the number of attack traffic also increases, which affects the

Table 1 Relationship of log file size with no. of attackers and traffic volume

\begin{tabular}{lll}
\hline File size (MB) & No. of attackers & Traffic vol. \\
\hline 10 & 100 & $0.24 \mathrm{~GB}$ \\
50 & 500 & $0.67 \mathrm{~GB}$ \\
100 & 1500 & $1.67 \mathrm{~GB}$ \\
200 & 2000 & $3.23 \mathrm{~GB}$ \\
400 & 4000 & $5.91 \mathrm{~GB}$ \\
600 & 6000 & $9.14 \mathrm{~GB}$ \\
800 & 8000 & $12.37 \mathrm{~GB}$ \\
1000 & 10,000 & $15.83 \mathrm{~GB}$ \\
\hline
\end{tabular}

mapper and reducer operation frequency and time. In short, with the increase in file size, the detection time increases and it will also increase the detection rate or the number of attackers IPs, which is a plus point.

Increase in cluster size hardly affects the detection time for files less than $400 \mathrm{MB}$ in size; on the contrary, in some cases, it might increase a little due to added management cost. Hadoop enables parallelism by splitting the files into different blocks of specified size. Files smaller than the Hadoop block size are not split over multiple nodes for execution. Therefore, the overall detection time remains the same over different cluster node.

Starting from the file size of $400 \mathrm{MB}$, the detection time improves with the increase of cluster size. For bigger files like $800 \mathrm{MB}$ and $1000 \mathrm{MB}$, Hadoop works more efficiently. We can see that the detection time reduces around 27 to $37 \mathrm{~s}$ for 800 and $1000 \mathrm{MB}$ files respectively, when the cluster size is increased from 2 to 10 nodes. This is because with $1000 \mathrm{MB}$ file, there are nine blocks, and with the increase in cluster size, Hadoop will assign the task to different nodes in parallel.

We have evaluated our detection phase on the basis of two different attack volumes: (1) 60-40 attack volume and (2) 80-20 attack volume. The main difference between these two volumes is that in $80 \%$ attack volume, the total time for detection slightly increases due to increase in the number of reducer operations. In $80 \%$ attack volume, the number of unique IPs is more than $60 \%$ attack volume which slightly increases (couple of seconds only) the reducer time for $80 \%$ attack volume file size. Again, this difference is only visible for larger log files.

Figure 10 shows the detection time on Hadoop cluster with a threshold value of 1000 . In this experiment, we only change the threshold value and all the remaining settings are similar to the Fig. 9. With the increase in threshold value, the total number of inputs for reducers also increases and this will increase the reducer time. This is the reason why majority of the results shown in Fig. 10 has couple of second higher detection time as compared to the results in Fig. 9.

\subsection{Effect of different block sizes}

Figure 11 show the effect of varying block sizes on the detection time for $1 \mathrm{~GB}$ file. In this experiment, we use fix threshold of 500, 80-20 attack volume, and three different blocks of size 32, 64, and $128 \mathrm{MB}$. For 1 GB file the block size of $128 \mathrm{MB}$ gives the maximum performance gains in terms of detection time with the increase in cluster nodes. With smaller block size, there are more splits, resulting in multiple tasks being schedule on a mapper and adds management overhead.

The effect of cluster size is prominent on large files. This is because with large files, Hadoop can effectively split the files in multiple blocks and distribute on the available 


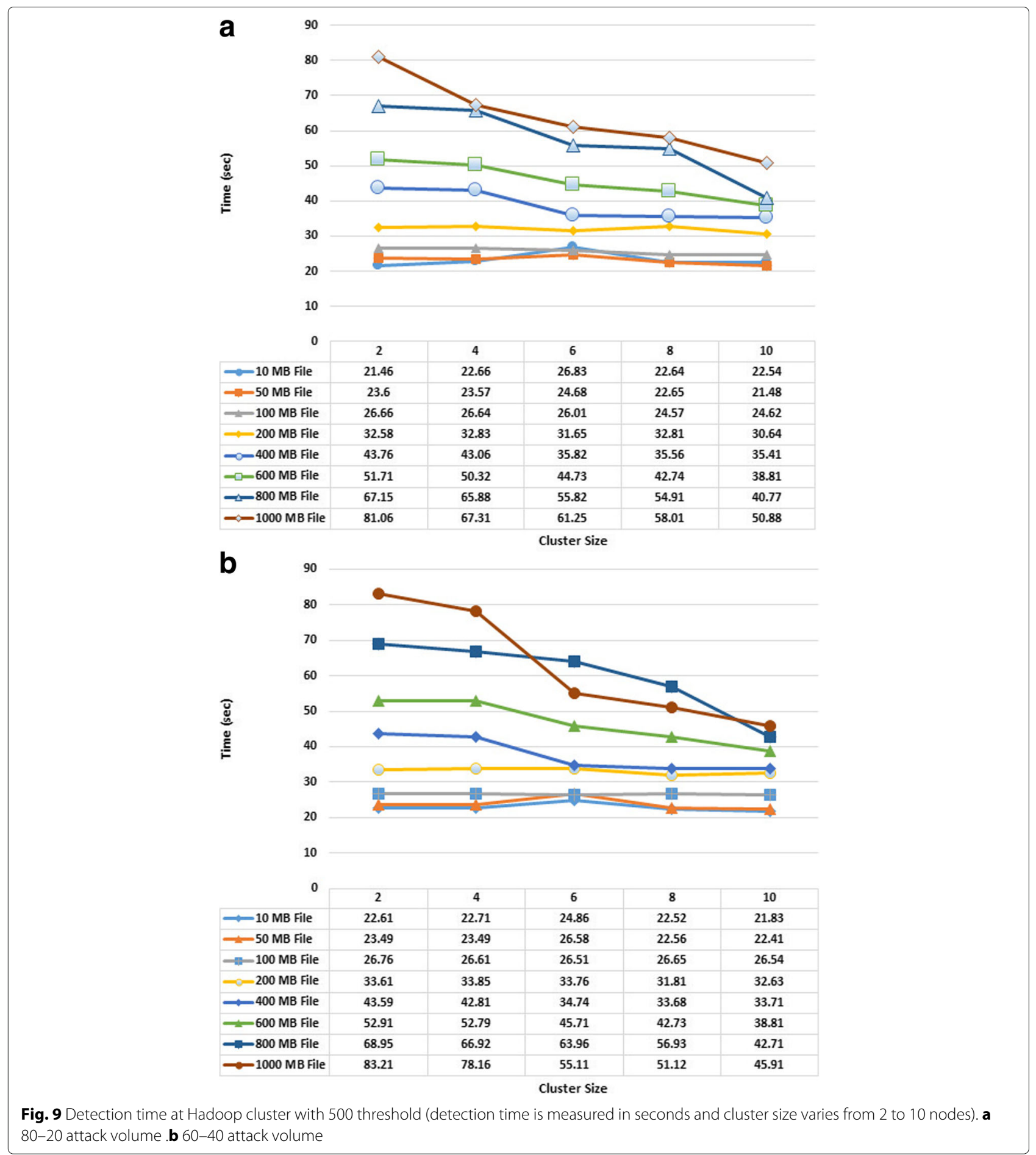

cluster nodes. Figure 12a and 12b show the effect of different block size and cluster node on the detection time for $10 \mathrm{~GB}$ and $20 \mathrm{~GB}$ respectively, with a fix threshold of 500 and $80-20$ attack volume. One hundred twentyeight megabyte block size gives the most efficient results; this is because when the number of blocks increases, the resource manager in Hadoop needs to manage each of the blocks and its result. Thus, it will take more time to manage each task. For larger block size, there is only one map task to process the whole large block. On a $10 \mathrm{~GB}$ file with a block size of $128 \mathrm{MB}$, Hadoop finished the detection task in approx. $7.5 \mathrm{~min}$ with a cluster size of 2 nodes. The detection time goes down to approx $4.5 \mathrm{mins}$ when the cluster size is increased to 10 nodes. For $20 \mathrm{~GB}$ 


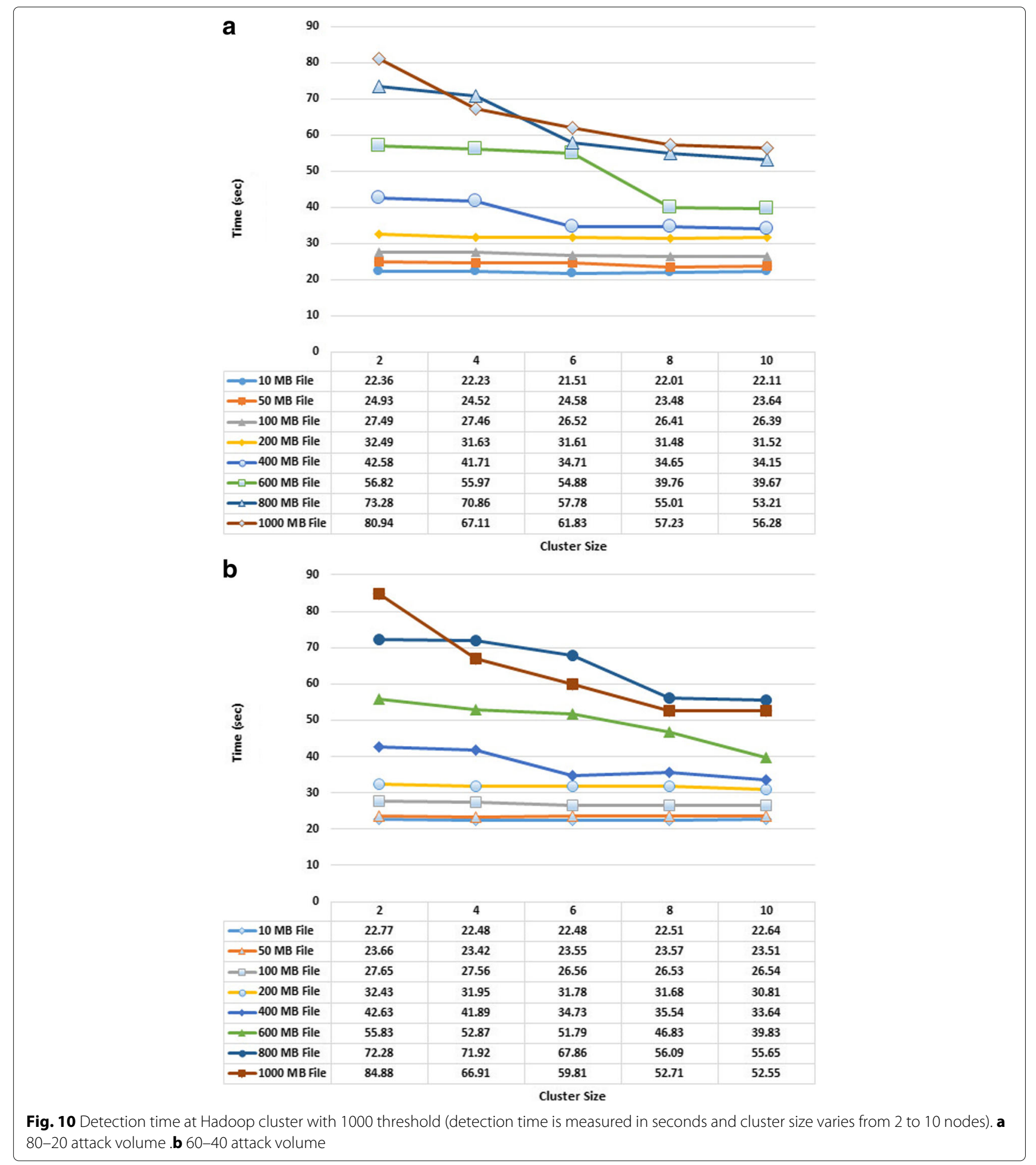

file with a block size of $128 \mathrm{MB}$, the time to finish the detection task is $14.6 \mathrm{~min}$ and $8.3 \mathrm{mins}$ on a cluster of 2 and 10 nodes respectively. If we approximate the numbers in Table 1, HADEC can effectively resolve $100 \mathrm{~K}$ attackers for an aggregate traffic volume of $159 \mathrm{GBs}$ with $10 \mathrm{~GB}$ of $\log$ file in just $4.5 \mathrm{~min}$. These numbers are doubled for $20 \mathrm{~GB}$.

\subsection{Overall framework performance}

Figures 13 and 14 show the overall performance of our proposed framework to detect the DDoS attacks. These numbers present the total time required for capturing, processing, transferring, and detection with different file sizes. For the experiments in Fig. 13a, we have used 80 20 attack volume, $128 \mathrm{MB}$ block size, and 500 threshold. 


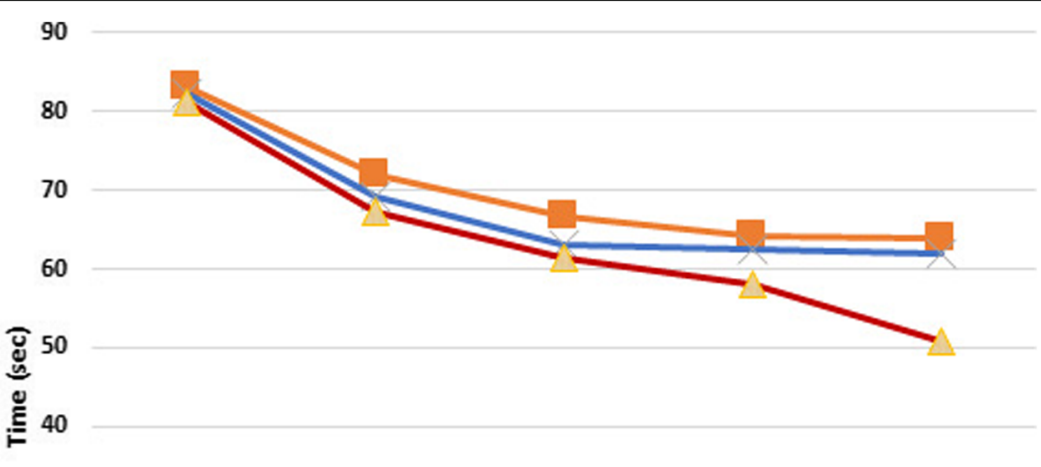

30

20

10

\begin{tabular}{|c|c|c|c|c|c|}
\hline 0 & 2 & 4 & 6 & 8 & 10 \\
\hline- Block Size 32 & 83.27 & 71.89 & 66.76 & 64.11 & 63.93 \\
\hline$\longrightarrow$ Block Size 64 & 82.33 & 69.08 & 63.02 & 62.63 & 61.9 \\
\hline - Block Size 128 & 81.06 & 67.3 & 61.25 & 58.01 & 50.88 \\
\hline
\end{tabular}

Fig. 11 Detection time with different block sizes on 1 GB file (detection time is measured in seconds and cluster size varies from 2 to 10 nodes)

For the experiments in Fig. 14a, we have only changed the threshold to 1000. The experiments in Fig. 13b and Fig. 14b used $60-40$ attack volume with 500 and 1000 threshold respectively.

In Fig. 13a, we can observe that with the increase in the file size, the overall overhead of capturing and transferring phase increases. A $10 \mathrm{MB}$ file takes approx. $16 \mathrm{~s}$ $(42 \%)$ in capturing/transferring phase and $21 \mathrm{~s}$ in detection phase. The best case of $1 \mathrm{~GB}$ file (10 node cluster) takes $178 \mathrm{~s}(77 \%)$ in capturing/transferring phase and just $50 \mathrm{~s}$ in detection phase. On the whole, it takes somewhere between 4.3 and 3.82 min to analyze $1 \mathrm{~GB}$ of $\log$ file that can resolve $10 \mathrm{~K}$ attackers and generated from an aggregate attack volume of 15.83 GBs. The variation of threshold and attack volume in Fig. 14a, Fig. 13b, and Fig. 14b slightly effects the overall detection time with couple of seconds.

\subsection{System benchmarks}

In this section, we evaluated the system benchmarks (CPU and memory usage) for the capturing, transferring, and detection phases in HADEC and log the readings every second. For the experiments, we used fix Hadoop block of $128 \mathrm{MB}$, threshold of 500 , and varied file sizes (10, 50, 100, 200, 400, 600, 800, and $1000 \mathrm{MB}$ ) with different number of cluster nodes. For the benchmark evaluations, we ran all the phases (capturing, transfer, and detection) in isolation to capture the performance of each phase without any external influence. Each phase shows a different CPU and memory usage pattern (see Table 2 for average usage). However, we observed that varying the experimental parameters like file size made no clear difference on CPU and memory usage within each phase (capturing, transfer, and detection). Thus, plotting CPU and memory usage for all the different file sizes results in overlapping plots that are illegible, confusing, and hard to analyze. The following sections discuss average system benchmarks during each phase of the proposed framework.

\subsubsection{Capture phase benchmarks}

The CPU measurement results show that there is no clear difference of varying file size on the CPU usage. The CPU usage largely remains between the range of 20 to $80 \%$ with an average of $50 \%$.

The memory usage remained constant at around $1000 \mathrm{MB}$ mark which is approximately $12-13 \%$ of the available memory.

The length of the experiments depends on the size of the file being captured. Once the required size is achieved, the capturing process stops and the CPU and memory usage smooths out within couple of seconds.

\subsubsection{Transfer phase benchmarks}

During the file transfer, the CPU usage is much lower than the capturing phase. The CPU usage mainly remains 
a

\section{4}

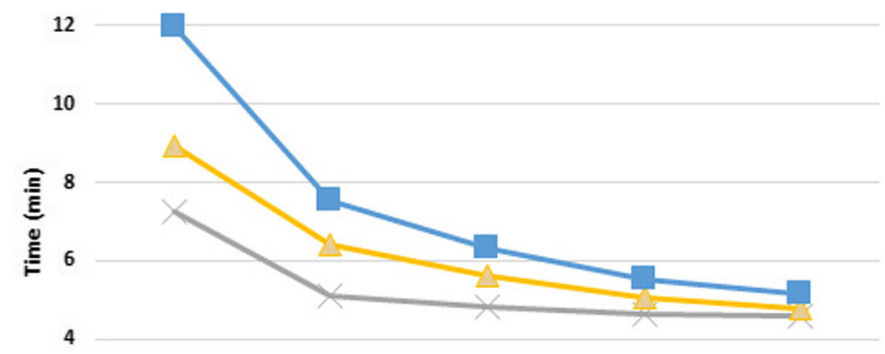

2

\begin{tabular}{|c|c|c|c|c|c|}
\hline 0 & 2 & 4 & 6 & 8 & 10 \\
\hline Block Size 32 & 7.25 & 5.11 & 4.81 & 4.66 & 4.58 \\
\hline -Block Size 64 & 8.94 & 6.42 & 5.61 & 5.07 & 4.8 \\
\hline$-=$ Block Size 128 & 11.95 & 7.53 & 6.32 & 5.53 & 5.17 \\
\hline
\end{tabular}

b

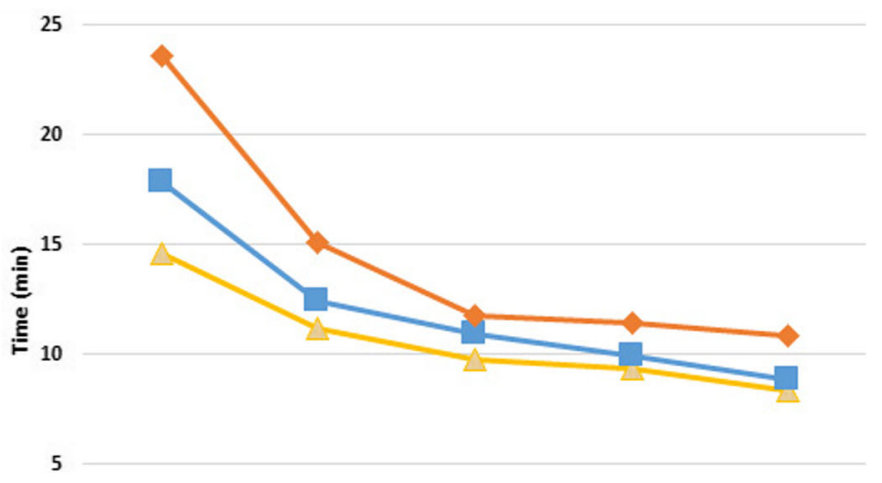

\begin{tabular}{|c|c|c|c|c|c|}
\hline 0 & 2 & 4 & 6 & 8 & 10 \\
\hline- Block Size 32 & 14.61 & 11.17 & 9.79 & 9.38 & 8.35 \\
\hline- -Block Size 64 & 17.84 & 12.42 & 10.95 & 9.94 & 8.89 \\
\hline —Block Size 128 & 23.61 & 15.11 & 11.81 & 11.42 & 10.86 \\
\hline
\end{tabular}

Fig. 12 Effect of block size on huge files (detection time is measured in minutes and cluster size varies from 2 to 10 nodes). a 10 GB file. b 20 GB file

between the range of 10 to $25 \%$ with an average of $15 \%$. When it comes to memory usage, the transfer phase consumes an average of $7600 \mathrm{MBs}$ which is approximately $94 \%$ of the available memory.

The length of the experiments depends on the size of the file being transferred to the detection server. Once the file is completely transferred, the CPU and memory usage smooths out within couple of seconds.

\subsubsection{Detection phase benchmarks at the Hadoop NameNode}

For the benchmarks at the detection phase, we varied the number of Hadoop cluster nodes for different file sizes and observed the CPU and memory usage at the NameNode.
The memory usage remained the same in overall detection phase, and on average, the memory usage remained between 85 and $95 \%$. The CPU usage on average remained below $40 \%$. In NameNode, we observed that with the increase in cluster size, the CPU usage decreases. Further, we also observed that when parallelism occurs in Hadoop due to bigger file size, the NameNode CPU usage become lower.

\section{Comparison with existing work and optimization recommendations}

This papers uses destination-based approach due to easy and cheaper DDoS detection with high accuracy. We leverage Hadoop to solve the scalability issue to detect multiple attacks from a huge volume of traffic by parallel 


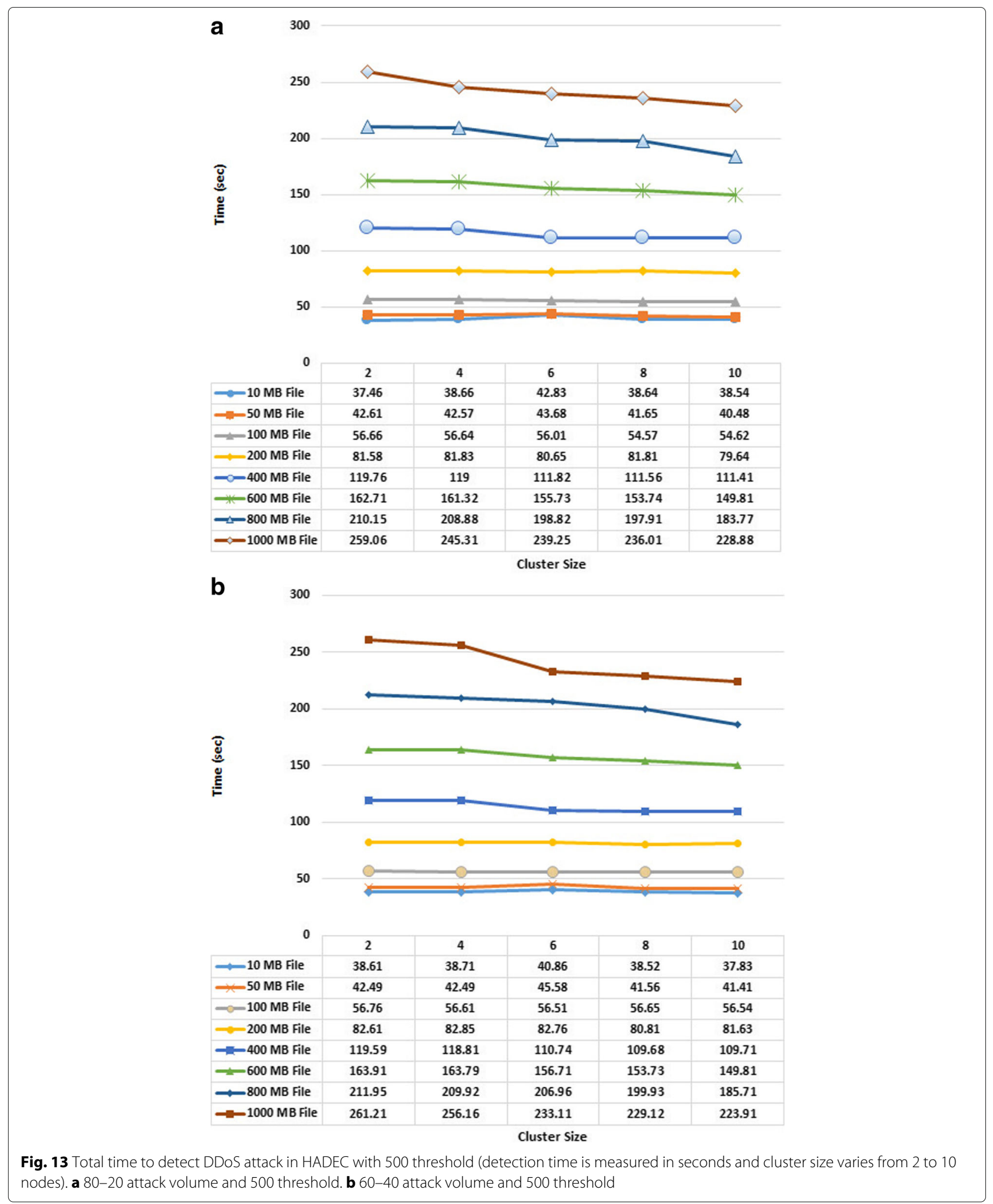

data processing. Traditional computational architectures mainly deploy single host-based approaches and enhance memory efficiency or customize process complexity.
Initially, Lee and Lee [27] proposed to use Hadoop for DDoS detection in a position paper. However, they only performed offline analysis on the traffic logs and their 


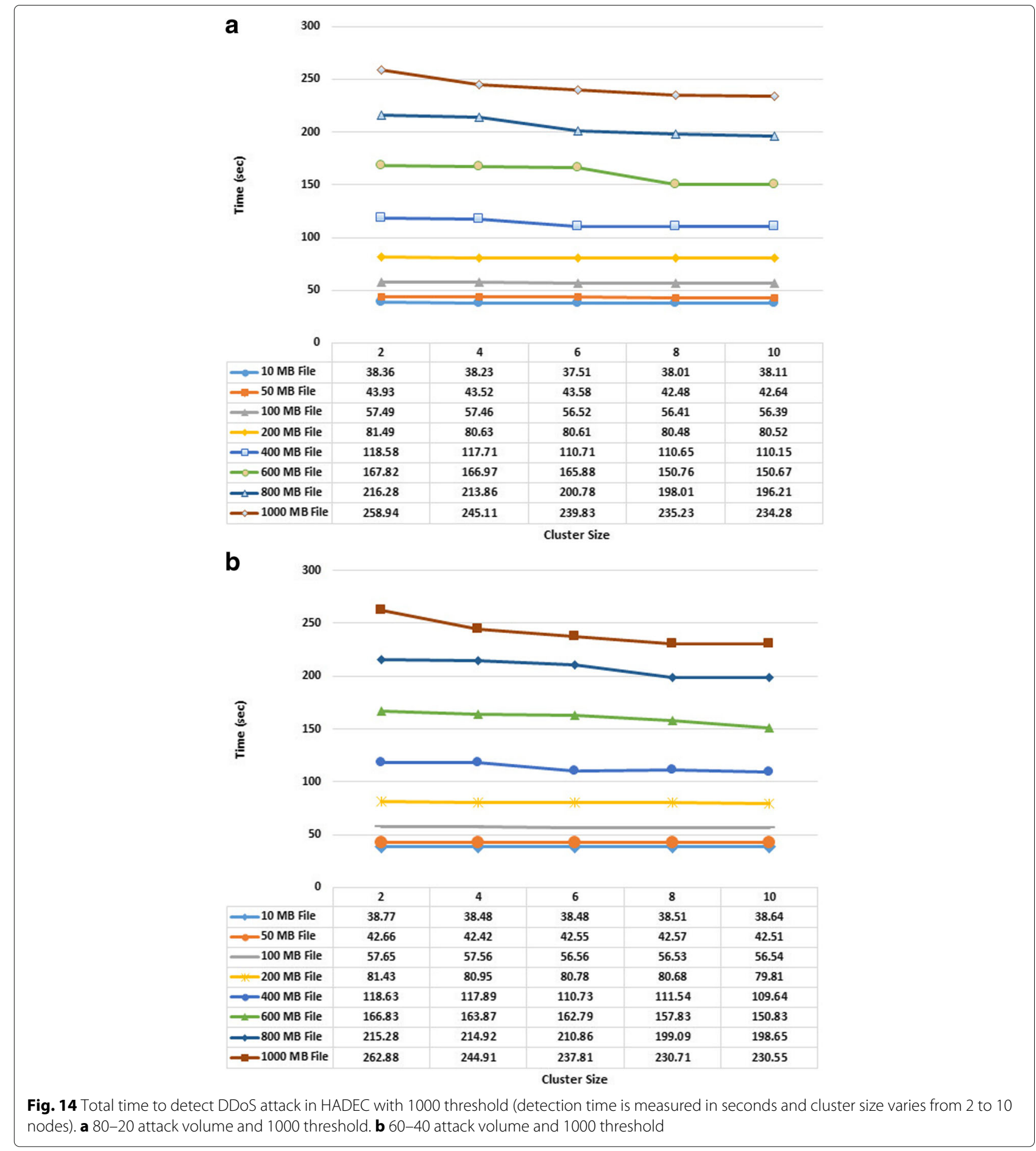

framework was also discussed like a blackbox. We covered all the design and implementation aspects and thoroughly evaluated live network to measure the efficacy of using Hadoop against DDoS. This is the first effort that prototyped a complete framework against flooding DDoS using Hadoop, and we extensively evaluated it as well.
The primary motivation behind this work was to present a framework based on distributed architecture that can handle high volume flooding attacks using lowend commodity hardware. This will ease the burden on small/medium enterprises and financial institute to deploy in-house cheap defenses and essentially save huge costs incurred from the third party DDoS mitigation 
Table 2 System benchmarks (average CPU and memory usage) during capture, transfer, and detection phases

\begin{tabular}{lll}
\hline Phase & CPU usage \% & Memory usage (\%) \\
\hline Capture phase & 50 & 13 \\
Transfer phase & 15 & 94 \\
Detection phase at NameNode & 40 & 90 \\
\hline
\end{tabular}

service providers. The testbed of our framework is divided into capturing and detection (Hadoop) server. This is a design choice to deploy HADEC on low-end machines (just core i5 with $8 \mathrm{~GB}$ ram) for real efficacy. For evaluations, we used desktop machines (core i5) with low computational power. Unlike [27], we extensively evaluated our frame by varying different parameters like log file size, attack volume, Hadoop cluster size, Hadoop splits or block size, and threshold for counter-based algorithm, and measured their impact on the performance of HADEC (like execution time and system benchmarks). These evaluation gave us insights on the strength and bottlenecks of the proposed framework and using this information system admin can customize their deployment strategy for optimal results.

Based on the results presented in this paper, we can conclude that HADEC is capable of analyzing high volume of DDoS flooding attacks in scalable manner. Several gigabytes of attack traffic can be analyzed in couple of minutes (from capturing to detecting). This also involves the bootstrapping time, i.e., initially when traffic was being captured the detection was idle. For an ongoing attack, both phases (capturing and detection) will be executing simultaneously and detection would be more effective. By using small size for log file, the overall detection time can be reduced to couple of seconds (30-40 s). But small log files also have an inherent limitation to the number of attacker's they can track. There is no single recommended setting; the admin will have to tweak the framework configuration that best match their requirement. We also noticed that with smaller files, Hadoop does not provide parallelism. This means that if any admin configures HADEC to work on small files of under $400 \mathrm{MB}$, there will be no point in setting up multiple node cluster. A single or two node clusters of Hadoop will do the job within few minutes (2-3) with the hardware settings we used in our testbed.

In our evaluations of HADEC, capturing and transferring phase showed the performance overhead and majority of the framework time was spent in these phases. This problem could be easily resolved by using reasonable to high-end server optimized for traffic operations, instead of mid-level core i5 desktop that are used in our testbed. A better approach, for banks and financial institutions, would be to deploy a network tap (custom monitoring and recording devices to capture and mirror network traffic) instead of a desktop system to capture and transfer the logs to detection server. A network tap with traffic mirroring capacity of 40-60 Gbps would range between 3000 and 4000 \$ (from different vendors like Gigamon, DataCom, Dualcom, etc.), and it would help transfer the log files representing $60 \mathrm{Gbps}(60 \mathrm{~Gb}$ or $7.5 \mathrm{GBs}$ will generate a log of approx. $550 \mathrm{MBs}$ ) of attack to the detection server in just matter of seconds and the attack logs can be further analyzed at the detection server within a minute. This cost effective (under $4 \mathrm{~K}-6 \mathrm{~K} \$$ ) solution would serve the defense needs of medium enterprises and financial institutions and help them save subscription cost to third party DDoS Mitigation services like Akamai Prolexic. Thus, a more effective and cost efficient deployment would be to use only couple of cluster nodes and configure a Network Tap as per traffic requirement for packet capturing and processing.

\section{Conclusions}

In this paper, we present HADEC, a scalable Hadoopbased live DDoS detection framework that is capable of analyzing DDoS attacks in affordable time. HADEC captures live network traffic, processess it to log relevant information in brief form, and uses MapReduce and HDFS to run detection algorithm for DDoS flooding attacks. HADEC solves the scalability, memory inefficiency, and process complexity issues of conventional solution by utilizing parallel data processing promised by Hadoop. The evaluation results showed that HADEC would need less than 5 min to process (from capturing to detecting) $1 \mathrm{~GB}$ of log file, generated from approx. 15.83 GBs of live network traffic. With small log file, the overall detection time can be further reduced to couple of seconds.

Based on the system benchmarks, we conclude that packet capturing is more CPU intensive as compared to $\log$ transfer phase. On the other hand, data log transfer consumes way more memory than the data capture phase. Further, the increase in cluster size and parallelism ease out the CPU usage at the Hadoop NameNode. We have observed that capturing of live network traffic incurs the real performance overhead for HADEC in terms of time. In worse case, the capturing phase consumes $77 \%$ of the overall detection time. As a future work, HADEC framework may allow potential optimizations to improve the capturing efficiency.

\section{Endnote}

${ }^{1}$ There is no silver bullet when it comes to DDoS defense. There are other forms of sophisticated DDoS attacks in the wild like reflected attacks, drive by attacks, resource attacks, flash-crowds, etc. However, this paper focus on designing and developing a Hadoop-based live 


\section{DDoS detection framework with flooding attacks as a proof of concept example. This framework will help to build similar systems for other attack types.}

\section{Acknowledgements}

We would like to thank the CS department of NUCES for providing us the testbed infrastructure. Special thanks to Mr. Talha Shamim Jafri (network manager) for his continuous help and support to keep the lab up and running.

\section{Authors' contributions}

Both authors have made substantial intellectual contribution from design, architecture, testbed deployment, evaluations, and writeup of this manuscript. Both authors read and approved the final manuscript.

\section{Authors' information}

Sufian Hameed received MSc in Media Information from RWTH-Aachen, Germany, in 2008 and received PHD in Information Security from George August University Goettingen, Germany in 2012. He currently hold a position of Assistant Professor in Department of Computer Science, National University of Computer and Emerging Sciences, Pakistan. His research interests revolve around all aspects of applied Computer Security, with an emphasis on network security, web security, mobile security, and secure architectures and protocols for Cloud and loTs.

Usman Ali started his education in Computer Science from COMSATS

University, Pakistan, from where he received his BS(CS) in the 2012. He did his MS-CS from National University of Computer and Emerging Sciences in 2015. His area of research interest is Machine Learning and Big Data Analysis. He is currently working as Software Engineer in Journey Xp.

\section{Competing interests}

There are no non-financial competing interests (political, personal, religious, ideological, academic, intellectual, commercial or any other) to declare in relation to this manuscript.

\section{Publisher's Note}

Springer Nature remains neutral with regard to jurisdictional claims in published maps and institutional affiliations.

\section{Received: 27 September 2017 Accepted: 12 July 2018 \\ Published online: 31 July 2018}

\section{References}

1. Dyn cyberattack. www.theguardian.com/technology/2016/oct/26/ddosattack-dyn-mirai-botnet. Accessed 09 July 2018

2. Github ddos attack. www.wired.com/story/github-ddos-memcached/. Accessed 09 July 2018

3. Hadoop. https://hadoop.apache.org/. Accessed 09 July 2018

4. Hadoop yarn. http://hortonworks.com/hadoop/yarn/. Accessed 09 July 2018

5. Iperf: network performance measurement tool. https://iperf.fr/. Accessed 09 July 2018

6. Loic: a network stress testing application. http://sourceforge.net/projects/ loic/. Accessed 09 July 2018

7. Mapreduce. http://wiki.apache.org/hadoop/MapReduce. Accessed 09 July 2018

8. Mausezahn. https://github.com/uweber/mausezahn. Accessed 09 July 2018

9. Operation Payback cripples MasterCard site in revenge for WikiLeaks ban, dec. 8, 2010. http://www.guardian.co.uk. Accessed 09 July 2018

10. Powerful attack cripples internet, oct. 23, 2002. http://www.greenspun. com/. Accessed 09 July 2018

11. Scapy. http://www.secdev.org/projects/scapy/. Accessed 09 July 2018

12. Secure copy. http://linux.die.net/man/1/scp. Accessed 09 July 2018

13. Tshark: network analyzer. www.wireshark.org/docs/man-pages/tshark. html. Accessed 09 July 2018

14. Yahoo on trail of site hackers, wired.com, Feb. 8, 2000. http://www.wired. com/. Accessed 09 July 2018

15. AA Cárdenas, PK Manadhata, SP Rajan, Big Data Analytics for Security. IEEE Security \& Privacy. 11(6), 74-76 (2013). https://doi.org/10.1109/MSP.2013. 138
16. B Claise, Cisco systems netflow services export version 9, rfc 3954 (informational) (2004)

17. PJ Criscuolo, Distributed denial of service: Trin00, tribe flood network, tribe flood network 2000, and stacheldraht ciac-2319 (2000). Technical report, DTIC Document

18. R Fontugne, J Mazel, K Fukuda, in 2014 IEEE Conference on Computer Communications Workshops (INFOCOM WKSHPS). Hashdoop: A MapReduce framework for network anomaly detection, (Toronto, 2014), pp. 494-499. https://doi.org/10.1109/INFCOMW.2014.6849281

19. J Francois, S Wang, W Bronzi, R State, T Engel, in 2011 IEEE International Workshop on Information Forensics and Security. BotCloud: Detecting botnets using MapReduce, (Iguacu Falls, 2011), pp. 1-6. https://doi.org/ 10.1109/WIFS.2011.6123125

20. J Francois, S Wang, R State, T Engel, ed. by J Domingo-Pascual, P Manzoni, S Palazzo, A Pont, and C Scoglio. NETWORKING 2011, volume 6640 of Lecture Notes in Computer Science (Springer, Berlin Heidelberg, 2011), pp. 1-14

21. S Hameed, U Ali, in IEEE/IFIP Network Operations and Management Symposium (NOMS). Efficacy of Live DDoS Detection with Hadoop, (Istanbul, 2016), pp. 488-494. https://doi.org/10.1109/NOMS.2016. 7502848

22. S Hameed, HA Khan, in International Conference on Networked Systems (NetSys). Leveraging SDN for collaborative DDoS mitigation, (Gottingen, 2017), pp. 1-6. https://doi.org/10.1109/NetSys.2017.7903962

23. S Hameed, UM Ali, On the efficacy of live ddos detection with hadoop. CoRR (2015). abs/1506.08953, arxiv.org/abs/1506.08953

24. S Hameed, HA Khan, SDN based collaborative scheme for mitigation of DDoS attacks. Futur. Internet. 23(2018)

25. C Kolias, G Kambourakis, A Stavrou, J Voas, DDoA in the loT: Mirai and other botnets. Computer. 50(7), 80-84 (2017)

26. Y Lee, W Kang, Y Lee, in Traffic Monitoring and Analysis, volume 6613 of Lecture Notes in Computer Science, ed. by J Domingo-Pascual, Y Shavitt, and S Uhlig. A hadoop-based packet trace processing tool (Springer, Berlin Heidelberg, 2011), pp. 51-63

27. Y Lee, Y Lee, in In Proceedings of The ACM CONEXT Student Workshop (CONEXT'11 Student). Detecting DDoS attacks with Hadoop (ACM, New York, 2011). Article 7, 2 pages. https://doi.org/10.1145/2079327.2079334

28. J Mirkovic, G Prier, P Reiher, in Proceedings of the 10th IEEE International Conference on Network Protocols (ICNP). Attacking DDoS at the source, (2002), pp. 312-321. https://doi.org/10.1109/ICNP.2002.1181418

29. J Mirkovic, G Prier, P Reiher, in Second IEEE International Symposium on Network Computing and Applications (NCA). Source-end DDoS defense, (2003), pp. 171-178. https://doi.org/10.1109/NCA.2003.1201153

30. K Park, H Lee, in Proceedings IEEE Conference on Computer Communications (INFOCOM). On the effectiveness of probabilistic packet marking for IP traceback under denial of service attack, vol. 1, (Anchorage, 2001), pp. 338-347. https://doi.org/10.1109/INFCOM.2001.916716

31. K Park, H Lee, On the effectiveness of route-based packet filtering for distributed DoS attack prevention in power-law internets. ACM SIGCOMM computer communication review. 31(4) (2001)

32. $V$ Paxson, Bro: A system for detecting network intruders in real-time. Comput. Netw. 31(23-24), 2435-2463 (1999)

33. A Pras, JJ Santanna, J Steinberger, A Sperotto, in International GI/ITG Conference on Measurement, Modelling, and Evaluation of Computing Systems and Dependability and Fault Tolerance. Ddos 3.0-how terrorists bring down the internet (Springer, 2016), pp. 1-4

34. S Ranjan, R Swaminathan, M Uysal, A Nucci, E Knightly, DDos-shield: DDos-resilient scheduling to counter application layer attacks. IEEE/ACM Trans. Networking (TON). 17(1), 26-39 (2009)

35. M Roesch, in Proceedings of the 13th USENIX Conference on System Administration, LISA '99. Snort - lightweight intrusion detection for networks (USENIX Association, Berkeley, 1999), pp. 229-238

36. JJ Santanna, et al., in IFIP/IEEE International Symposium on Integrated Network Management (IM). Booters - An analysis of DDoS-as-a-service attacks, (Ottawa, 2015), pp. 243-251. https://doi.org/10.1109/INM.2015. 7140298

37. H Wang, C Jin, KG Shin.Defense against spoofed IP traffic using hop-count filtering. IEEE/ACM Trans. Networking (ToN). 15(1), 40-53 (2007)

38. A Yaar, A Perrig, D Song, in Proceedings of IEEE Symposium on Security and Privacy (IEEE S\&P). Pi: a path identification mechanism to defend against 
DDoS attacks, (2003), pp. 93-107. https://doi.org/10.1109/SECPRI.2003. 1199330

39. X Yang, D Wetherall, $T$ Anderson, A DoS-limiting network architecture SIGCOMM Computer Communication Review. 35(4), 241-252 (2005). https://doi.org/10.1145/1090191.1080120

40. X Yang, D Wetherall, T Anderson, TVA: a DoS-limiting network architecture. IEEE/ACM Trans. Networking. 16(6), 1267-1280 (2008)

41. ST Zargar, J Joshi, D Tipper, A survey of defense mechanisms against distributed denial of service (DDoS) flooding attacks. Commun. Surv. Tutorials IEEE. 15(4), 2046-2069 (2013)

\section{Submit your manuscript to a SpringerOpen ${ }^{\circ}$ journal and benefit from:}

- Convenient online submission

- Rigorous peer review

- Open access: articles freely available online

- High visibility within the field

- Retaining the copyright to your article

Submit your next manuscript at $\gg$ springeropen.com 